\title{
Glutathione system in Wolfram syndrome 1-deficient mice
}

\author{
RANDO POROSK $^{1,2}$, KALLE KILK ${ }^{1,2}$, RIINA MAHLAPUU ${ }^{1,2}$, ANTON TERASMAA ${ }^{2,3}$ and URSEL SOOMETS ${ }^{1,2}$ \\ ${ }^{1}$ Department of Biochemistry, Institute of Biomedicine and Translational Medicine; \\ ${ }^{2}$ Centre of Excellence for Genomics and Translational Medicine; ${ }^{3}$ Department of Physiology, Institute \\ of Biomedicine and Translational Medicine, University of Tartu, 50411 Tartu, Estonia
}

Received April 1, 2017; Accepted July 27, 2017

DOI: $10.3892 / \mathrm{mmr} .2017 .7419$

\begin{abstract}
Wolfram syndrome 1 (WS) is a rare neurodegenerative disease that is caused by mutations in the Wolfram syndrome 1 (WFS1) gene, which encodes the endoplasmic reticulum (ER) glycoprotein wolframin. The pathophysiology of WS is ER stress, which is generally considered to induce oxidative stress. As WS has a well-defined monogenetic origin and a model for chronic ER stress, the present study aimed to characterize how glutathione (GSH), a major intracellular antioxidant, was related to the disease and its progression. The concentration of GSH and the activities of reduction/oxidation system enzymes GSH peroxidase and GSH reductase were measured in $W f s l$-deficient mice. The GSH content was lower in most of the studied tissues, and the activities of antioxidative enzymes varied between the heart, kidneys and liver tissues. The results indicated that GSH may be needed for ER stress control; however, chronic ER stress from the genetic syndrome eventually depletes the cellular GSH pool and leads to increased oxidative stress.
\end{abstract}

\section{Introduction}

Wolfram syndrome 1 (WS; OMIM: 222300) is a rare autosomal recessive neurodegenerative disease. It is characterized by DIDMOAD; that is, the development of diabetes insipidus, diabetes mellitus, optic atrophy and deafness $(1,2)$. WS is caused by mutations in the Wolfram syndrome 1 (WFS1) gene. WFS1 encodes wolframin, a transmembrane glycoprotein that is ubiquitously expressed, with the highest levels expressed in heart, brain, pancreatic $\beta$-cells, placenta and lungs. Wolframin is primarily located in the endoplasmic reticulum (ER) and $W f s l$-deficiency causes ER stress and cellular dysfunction $(1,2)$. Human WFS1 protein amino acid content is $87 \%$ conserved with the mouse homolog (Wfs1). Wfs1 comprises

Correspondence to: Mr. Rando Porosk, Department of Biochemistry, Institute of Biomedicine and Translational Medicine, University of Tartu, 19 Ravila, 50411 Tartu, Estonia

E-mail: rando.porosk@ut.ee

Key words: glutathione, Wolfram syndrome 1, Wolfram syndrome, wolframin, diabetes nine central transmembrane domains, an extracytoplasmic amino-terminus and an intracytoplasmic carboxyl-terminus; and nitrogen-linked glycosylation is essential for its biogenesis and stability (3). As an ER glycoprotein, wolframin may participate in membrane trafficking, regulation of $\mathrm{Ca}^{2+}$ homeostasis and protein processing (4). In patients with WS, pancreatic $\beta$-cell death may be associated with impaired $\beta$-cell dysfunction, as seen in patients with type 2 diabetes (5). The expression of $W f s l$ in mouse pancreatic islets was previously demonstrated to be upregulated during glucose-induced insulin secretion (6).

WS is related to different pathological conditions and it is a valuable disease model for identifying biomarkers associated with ER stress, juvenile-onset diabetes and neurodegeneration $(1,2)$. In addition, WS arises from mutations of a single gene; therefore, it may be a good model for investigating the mechanisms of ER dysfunction compared with multifactorial conditions like 'ordinary' type 1 and type 2 diabetes (7).

ER stress is a situation in which misfolded proteins accumulate in the lumen of ER; the unfolded protein response (UPR) that follows upregulates survival related signaling and chaperone synthesis, and inhibits the synthesis of many other proteins (8). Oxidative stress is considered to increase ER stress through the activity of oxidoreductin-1 and protein disulfide isomerases $(9,10)$. Protein folding depends on many factors other than chaperones; among these factors, proper reduction/oxidation (redox) ratio and glutathione (GSH) levels are directly and indirectly via GSH sensitive regulatory proteins involved in appropriate disulfide bridge formation (11). GSH is a thiol-containing tripeptide comprising $\gamma$-glutamate, cysteine and glycine, and its formation occurs in the cytosol and requires no folding compared with redox enzymes. ER stress is known to enhance GSH synthesis through the transcription factors cyclic AMP-dependent transcription factor 4 and nuclear factor erythroid 2-related factor $2(12,13)$. Although the aim of UPR is cell survival, persistent stress may induce the cells to trigger apoptosis. It remains unknown how acute and chronic ER stress are managed by individual cells and organisms as a whole.

$\mathrm{GSH}$ is a potent scavenger of free radicals and other oxidant species in which it is oxidized by selenium-containing GSH peroxidase (GPx) to oxidized GSH (GSSG) and is reduced back to GSH by GSH reductase (GR). The antioxidant capacity of cells is mainly described by the GSSG/GSH redox ratio and has been related to several pathological states, 
including neurodegenerative, cardiovascular and immune system diseases (14). Therefore the present study aimed to characterize the GSH system in the heart, kidney, liver and pancreatic tissues of $W f s 1$-deficient mice.

\section{Materials and methods}

Chemicals. All chemicals were from Sigma-Aldrich (Merck KGaA, Darmstadt, Germany). The DC Protein Assay was from Bio-Rad Laboratories, Inc. (Hercules, CA, USA) and Glutathione Assay, Glutathione Peroxidase Assay and Glutathione Reductase Assay kits were from Cayman Chemical Company (Ann Arbor, MI, USA).

Animals and tissue collection. All animal experiments in this study were carried out in accordance with European Communities Directive (86/609/EEC) and the study was approved by The Estonian National Board of Animal Experiments (permit number 36; obtained July 23, 2014). Mice (weight, $22 \mathrm{~g}$ at 2 months old; $28 \mathrm{~g}$ at 6 months old) were housed under standard laboratory conditions: 12-h light/dark cycle (lights out at 07:00 PM) at $20 \pm 2^{\circ} \mathrm{C}, 40-60 \%$ humidity, with access to chow diet and water ad libitum. A total of 48 male (age 2 and 6 months) wild-type (WT; $W f s 1^{+/+}$) and $W f s 1$-deficient [heterozygous (HZ; Wf $s 1^{+/}$) and homozygous (knockout, $\mathrm{KO}$; Wfs $\left.1^{-/}\right]$mice were used throughout this study. The [(129S6/SvEvTac x C57BL/6) x (129S6/SvEvTac x C57BL/6)] F2 hybrids Wfs1-deficient mice were generated in the Institute of Biomedicine and Translational Medicine, University of Tartu (Tartu, Estonia) and the detailed generation, breeding and genotyping analysis of the mice was as described previously (15).

Mice were euthanized by manual cervical dislocation. Liver, heart, pancreas and kidney tissues were collected, perfused with ice-cold saline, snap frozen in liquid nitrogen and stored at $-80^{\circ} \mathrm{C}$ until processing. Each experimental group consisted of eight animals.

Sample preparation. Tissue samples (15-250 $\mathrm{mg})$ were homogenized in $0.1 \mathrm{M}$ phosphate buffer (1:10 w/v; pH 7.4) and centrifuged for $15 \mathrm{~min}$ at $10,000 \mathrm{x} \mathrm{g} 4^{\circ} \mathrm{C}$. Supernatants were collected and immediately aliquoted for the measurement of total GSH (tGSH) or the enzymatic activity of GR or GPx. For the measurement of tGSH and GSSG, proteins were precipitated with $10 \%$ metaphosphoric acid (1:1 w/v) to avoid interference owing to particulates and sulfhydryl groups in the assay.

Measurement of total and oxidized glutathione concentration. tGSH and GSSG levels in heart, liver, kidney and pancreas were measured using the Glutathione Assay kit according to manufacturer's protocol, which uses the optimized enzymatic GR recycling method first described by Tietze (16). Briefly, $50 \mu \mathrm{l} / \mathrm{ml}$ of $4 \mathrm{M}$ triethanolamine was added to the supernatant to increase the $\mathrm{pH}$ of the samples and the final sample volume was $50 \mu \mathrm{l}$ per well. Next, the thiol group of GSH in the sample (50 $\mu 1$ ) reacted with $450 \mu 1$ 5,5-dithiobis-(2-nitrobenzoic acid) (DTNB) resulting in the formation of the yellow-colored 5-thio-2-nitrobenzoic acid (TNB) and a mixed disulfide of GSH and DTNB after 25 min incubation. The latter is reduced by GR to recycle the GSH and produce extra TNB. The production of total TNB is directly proportional to the concentration of GSH in the sample. The quantification of GSSG is accomplished by first derivatizing GSH with $1 \mathrm{M}$ 2-vinylpyridine $(10 \mu \mathrm{l} / \mathrm{ml})$. The tGSH and GSSG samples were measured at $412 \mathrm{~nm}$ with a Tecan Sunrise spectrophotometer (Tecan Group, Ltd., Männedorf, Switzerland).

Measurement of the activity of GPx and GR. The mRNA or protein expression levels of an enzyme do not necessarily result in an increase in activity, therefore the activity of GPx and GR was measured with a Glutathione Peroxidase Assay Kit and a Glutathione Reductase Assay Kit according to the manufacturer's protocols, respectively. GR catalyzes the NADPH-dependent reduction of GSSG to GSH and therefore maintains adequate levels of cellular GSH. GPx catalyzes the reduction of hydrogen peroxide to protect the cell from oxidative stress and uses GSH as the ultimate electron donor. The assay measures GR activity by the rate of NADPH oxidation and GPx activity indirectly by coupled reaction with GR (17,18).

Statistical analysis. Data were analyzed using GraphPad Prism version 5.0.0 for Windows (GraphPad Software, Inc., La Jolla, CA, USA). The results are presented as the mean \pm standard error of the mean. Comparisons between groups were made using one-way analysis of variance followed by Tukey's test. $\mathrm{P}<0.05$ was considered to indicate a statistically significant difference.

\section{Results}

The concentration of tGSH and the activities of GPx and GR were measured in the heart, liver, kidneys and pancreatic tissue of $\mathrm{KO}, \mathrm{HZ}$ and WT mice.

Kidney. The 2-month-old KO mice exhibited a significantly lower level of tGSH concentration in the kidney tissue compared with tGSH concentration in the WT littermates (1.6-fold; $\mathrm{F}_{2,15}=5.9 ; \mathrm{P}<0.05$; Fig. 1A). HZ 6-month-old mice exhibited a 1.2-fold higher concentration of tGSH in the kidney tissue compared to WT mice $\left(\mathrm{F}_{2,19}=8.2 ; \mathrm{P}<0.05\right.$; Fig. 1B). The level of GSSG was below the detection limit and could not be measured. GPx activity was 1.7-fold higher in 2-month-old KO mice compared with WT mice $\left(\mathrm{F}_{2,21}=18.0 ; \mathrm{P}<0.0001\right.$; Fig. 2A). GR activity was 1.4 -fold higher in 2-month-old $\mathrm{KO}$ mice, but this was not indicted to be statistically significant (Fig. 2B). GPx and GR activities in 6-month-old mice could not be measured due to their insufficient activity in the available amount of renal tissue.

Heart. Analyses on heart tissues identified slightly lower, albeit not statistically significant, levels of GSSG and GSH (Fig. 3) compared with WT. In 6-month-old KO mice, the GSH concentration was 1.5 -fold lower $\left(\mathrm{F}_{2,20}=10.9 ; \mathrm{P}<0.001\right.$; Fig. 3) in the heart tissue compared with WT littermates. GPx activity was slightly higher and GR activity lower in KO 2-month-old mice compared with WT mice, but these differences were not statistically significant (Fig. 3). The GSSG/GSH ratio was 2 -fold higher $\left(\mathrm{F}_{2,20}=4.9 ; \mathrm{P}<0.05\right)$ in 

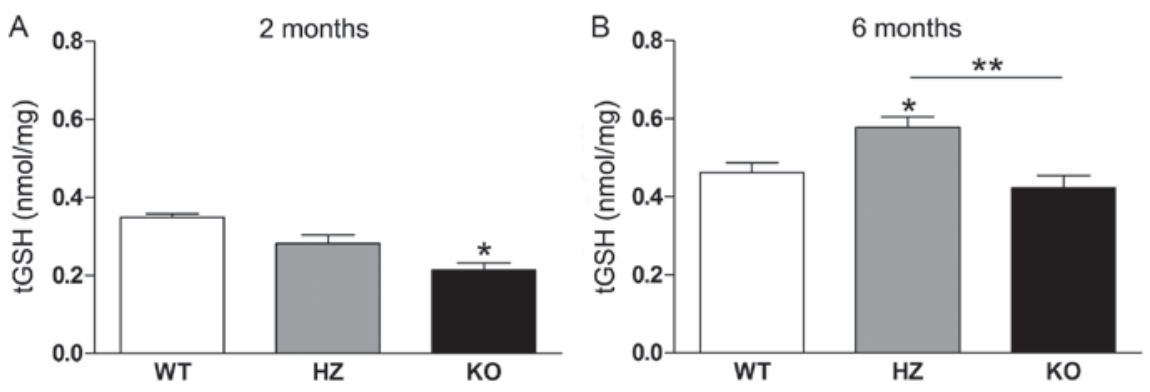

Figure 1. Concentration of tGSH in kidneys of Wfs $1 \mathrm{WT}$, and KO mice. tGSH concentrations were measured in the kidneys of the WT, HZ and KO (A) 2-month-old mice and in (B) 6-month-old mice. Data are presented as the mean \pm standard error of the mean; $n=8 /$ group; ${ }^{*} \mathrm{P}<0.05$ vs. WT; ${ }^{* *} \mathrm{P}<0.001$. HZ, heterozygous; KO, knockout; tGSH, total glutathione; Wfsl, Wolfram syndrome 1; WT, wild-type.
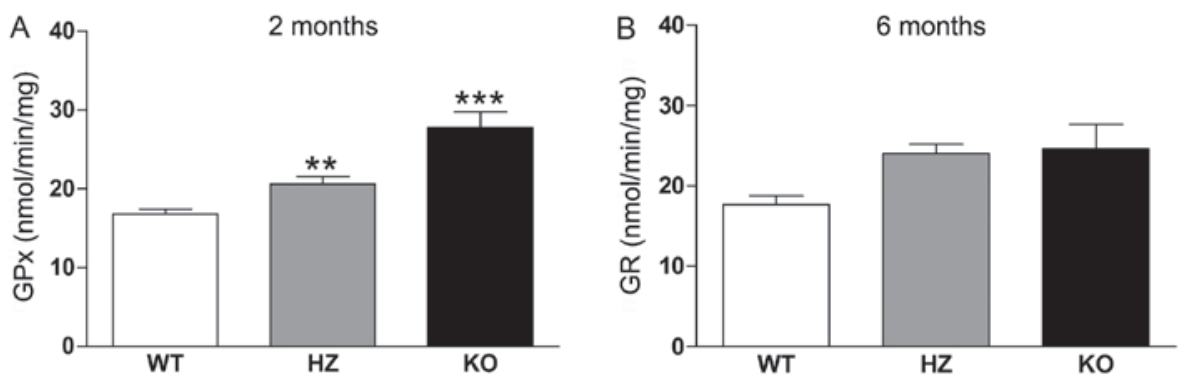

Figure 2. GPx and GR activity in kidneys of Wfsl WT, HZ and KO mice. 2-month old mouse kidneys were examined for (A) GPx and (B) GR activity. Data are presented as the mean \pm standard error of the mean; $\mathrm{n}=8 /$ group; ${ }^{* *} \mathrm{P}<0.001 ;{ }^{* * *} \mathrm{P}<0.0001$. GPx, glutathione peroxidase; GR, glutathione reductase; HZ, heterozygous; KO, knockout; WT, wild-type.

older and slightly higher in younger $W f s l$-deficient mice compared with WT (Fig. 4).

Liver. In the liver, there was a 1.1-fold higher level (not statistically significant) of GSH in 2-month-old KO mice and a 1.7-fold lower level of GSH in 6-month-old mice compared with WT littermates $\left(\mathrm{F}_{2,21}=4.2 ; \mathrm{P}<0.05\right.$; Fig. 5). GR activity was 1.6-fold lower in 2-month-old $\mathrm{KO}$ mice compared to WT mice $\left(\mathrm{F}_{2,21}=6.4 ; \mathrm{P}<0.001\right)$. Notably, the activity of GPx $\left(1.3\right.$-fold; $\left.\mathrm{F}_{2,21}=5.6 ; \mathrm{P}<0.05\right)$ and $\mathrm{GR}\left(1.6\right.$-fold; $\mathrm{F}_{2,20}=5.2$; $\mathrm{P}<0.05)$ were significantly increased in 6-month-old $\mathrm{KO}$ and HZ mice compared with WT littermates. These data indicated more intensive usage of GSH by GPx in older mice, whereas the activity of GR is recovered.

Pancreas. No significant differences were identified in the GSH system of the pancreas of Wfs 1 -deficient 2- and 6-month-old mice (Fig. 6A and B, respectively). GSSG levels were below the detection limit and could not be measured.

\section{Discussion}

Oxidative stress is strongly associated with ER stress; ER and oxidative stress reduce the GSH capacity and induce the synthesis of reduced GSH $(9,13)$. The present study examined the GSH system in WfsI-deficient mice to characterize the extent of oxidative stress in several tissues under chronic ER stress. The results indicated that the GSH system was not identical in all tissues of Wfsl-deficient mice. It is particularly complex for the heterozygous mice, in which the tGSH levels may be up- or downregulated depending on tissue type and age.
One of the highest expression levels of Wfsl is found in heart tissue (1). In the present study, WfsI deficiency also exhibited the greatest effects on the GSH system in the heart. At 2-months old, when the disease has not yet fully manifested its clinical symptoms $(1,3,6)$, the levels of both GSH and GSSG were slightly decreased in KO mice, but the GSSG/GSH ratio was increased, though not significantly. Enzyme levels favor the change in the ratio with a tendency of increased GPx activity and reduced GR activity. As the disease progresses, the changes become more significant. The $\mathrm{HZ}$ is between the WT and KO. A previous GPx-1-KO study has suggested that GPx may have anti-ER stress effects (19), and therefore its upregulation may also be part of the UPR (20). In addition to ER stress response, particularly at the older age (6 months), the GSH system may be altered by complications of systemic WS manifestations such as diabetes. In streptozotocin-induced diabetes, a decrease of GR activity in heart has been reported (21).

The pancreas is another organ with high levels of WfsI expression. Its exocrine and endocrine functions require active synthesis of proteins, and makes the pancreas particularly susceptible to the effects of $W f s l$ deficiency and UPR. Pancreatic $\beta$-cells have low levels of antioxidant enzyme expression and activity, including superoxide dismutases, catalase and GPx (22). By contrast, the catalytic subunit of $\gamma$-glutamylcysteine ligase, which is the rate-limiting enzyme for GSH biosynthesis, is highly expressed in pancreatic islet cells (23). The inability to properly process insulin is a key event in WS pathophysiology and the development of diabetes is triggered by the deficiency of insulin (24). In the present study, whole pancreas tissue was examined, making the 


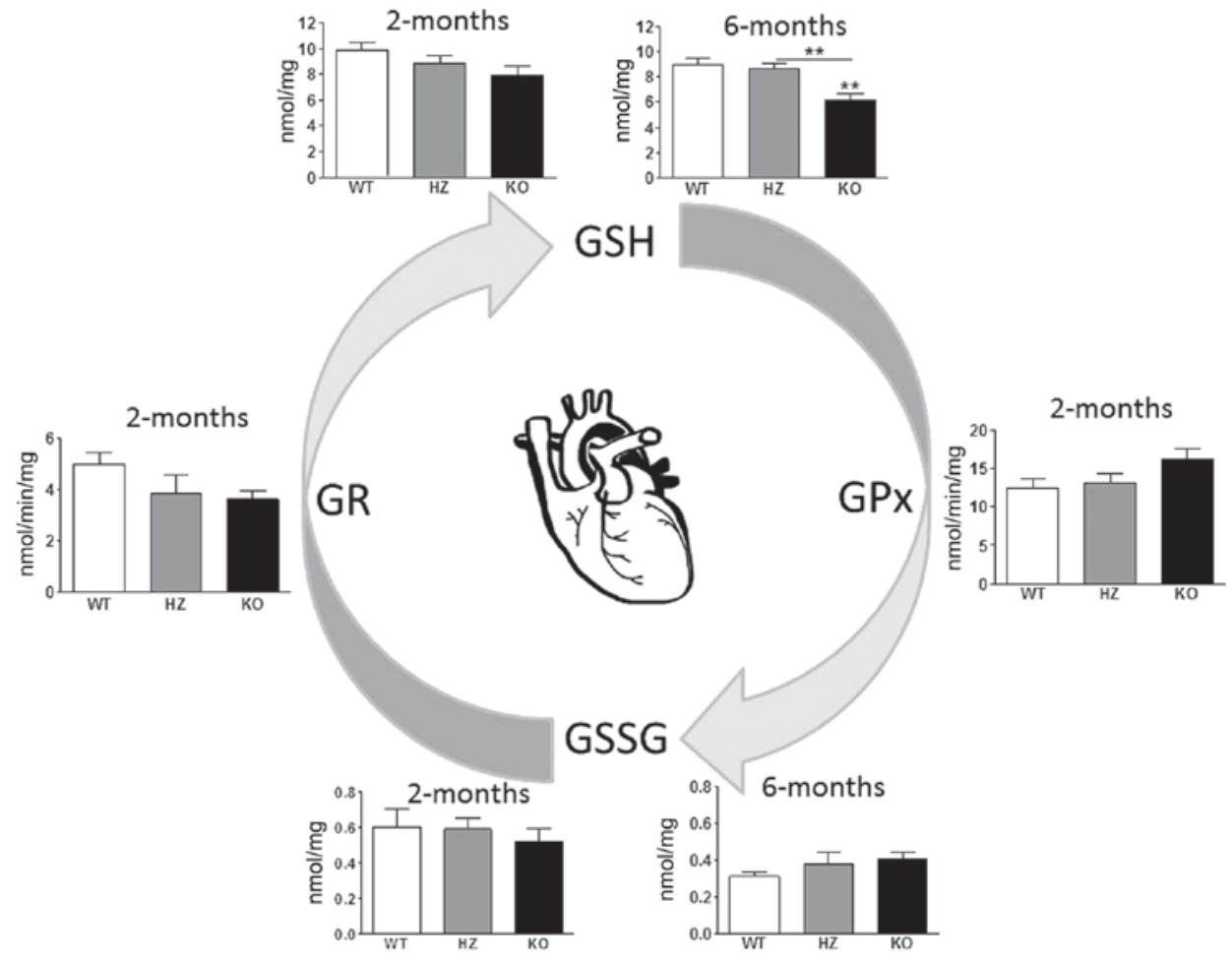

Figure 3. Schematic fig. of the glutathione system affected by Wfs 1 -knockout in 2- and 6-month-old mouse heart tissue. Data are presented as the mean \pm standard error of the mean; $\mathrm{n}=8 /$ group; ** $\mathrm{P}<0.001$. GPx, glutathione peroxidase; GR, glutathione reductase; GSH, reduced glutathione; GSSG oxidized glutathione; HZ, heterozygous; KO, knockout; Wfsl, Wolfram syndrome 1; WT, wild-type.

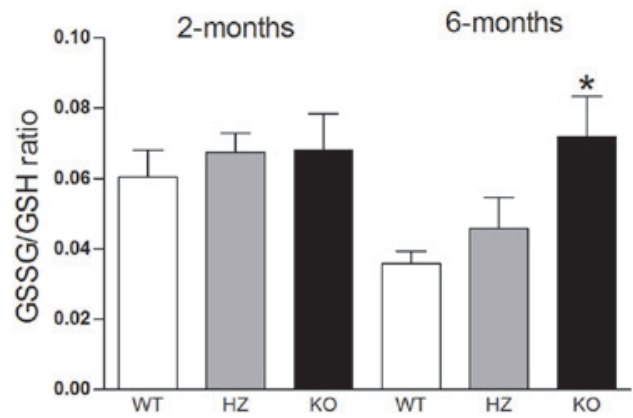

Figure 4. GSH redox ratio (GSSG/GSH) in the heart of 2- and 6-month-old Wfs 1 WT, HZ and KO mice. Data are presented as the mean \pm standard error of the mean; $\mathrm{n}=8$ /group; ${ }^{\mathrm{P}}<0.05$. GSH, reduced glutathione; GSSG oxidized glutathione; HZ, heterozygous; KO, knockout; Wfs 1 , Wolfram syndrome 1; WT, wild-type.

results more relevant for the larger exocrine function. With no identified significant changes in tGSH levels and with GSSG under the detection limit, oxidative stress is seemingly well controlled.

Liver has lower Wfsl expression compared with heart or pancreas (2). The liver serves a major role in the regulation of carbohydrate metabolism, such as maintaining the blood glucose level and homeostasis in general. An entire spectrum of liver diseases have been associated with type 2 diabetes, including abnormal liver enzymes, nonalcoholic fatty liver disease, cirrhosis, hepatocellular carcinoma and acute liver failure (25). A decrease in GSH levels in the diabetic liver and remarkable increment of GSSG/GSH ratio have been reported previously (26). In the present study, 2-month-old $W f s 1$-KO mice at young age, GSH expression similar to WT expression levels; at 2 months of age hyperglycemia and diabetes has not yet manifested itself in mouse models $(27,28)$, therefore changes from diabetic complications are not expected. At 6 months of age, however, the expected decrease was observed. A small GSH increase at young age could be expected in response to ER stress $(12,13)$, which at that time is not overwhelming the compensatory mechanisms. It may be considered that the heart has either stronger stress owing to higher dependency on $W f_{s} 1$ or its compensatory mechanisms are weaker, which may lead to a tendency of GSH reduction even early on. The activity of the two main GSH redox enzymes, GPx and GR, also suggest that the early and late liver tissues are experiencing different situations. Early on, GPx activity remains unchanged in KO mice compared with 'healthy' WT, although GR activity is reduced. At 6 months old, GR activity in $\mathrm{KO}$ mice returns to similar levels as WT (or even surpasses the WT activity in $\mathrm{HZ}$ ), but GPx activity was increased.

Similar to the liver, kidneys express $W f s l$ at low levels. A commonly observed complication of WS and type 2 diabetes is diabetic nephropathy, which is a frequent cause of mortality in diabetic patients (29). It has been postulated that oxidative stress may be a key component in the development of nephropathy (30). Chronic exposure to high levels of glucose leads to a decrease in GPx activity in vascular endothelial and kidney cells $(31,32)$. It has been demonstrated that exposure to oxidative stress inducers such as carbon tetrachloride increases GPx activity in rat kidneys (33). Rats treated with ethanol exhibited increases in both GPx and GR activity in kidneys (34). High glucose concentration has been reported to decrease $\gamma$-glutamylcysteine ligase 

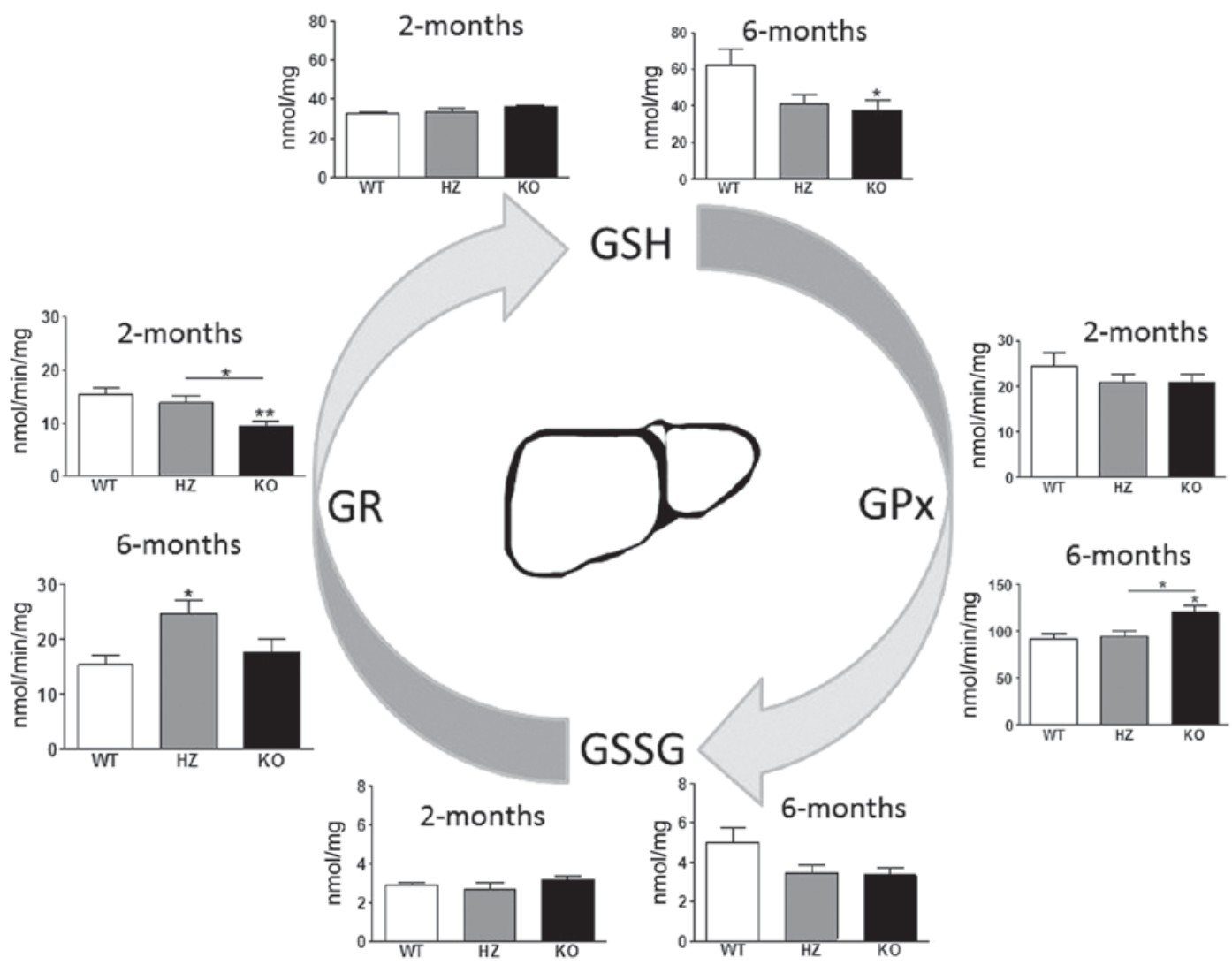

GSSG

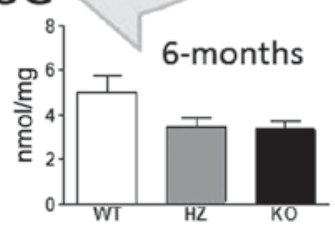

Figure 5. Schematic fig. of glutathione system affected by Wfs1-knockout in 2- and 6-month-old mice in liver tissue. Data are presented as the mean \pm standard error of the mean; $\mathrm{n}=8 /$ group; ${ }^{*} \mathrm{P}<0.05$ vs. WT; * $\mathrm{P}<0.001$ vs. WT. GPx, glutathione peroxidase; GR, glutathione reductase; GSH, reduced glutathione; GSSG oxidized glutathione; HZ, heterozygous; KO, knockout; Wfsl, Wolfram syndrome 1; WT, wild-type.
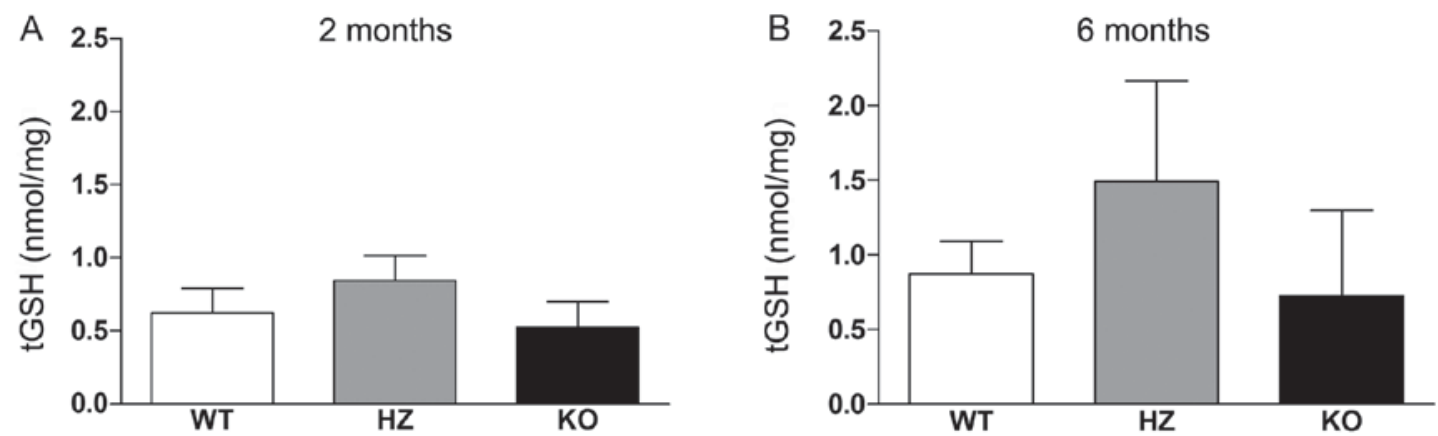

Figure 6. Concentration of tGSH in pancreas of Wfsl WT, HZ and KO mice. tGSH levels were measured in the pancreas of both (A) 2-month-old and (B) 6-month-old mice. Data are presented as the mean \pm standard error of the mean; $\mathrm{n}=8$ /group. HZ, heterozygous; KO, knockout; tGSH, total glutathione; Wfsl, Wolfram syndrome 1; WT, wild-type.

expression and GSH levels in mesangial cell culture (32). Therefore increased GPx activity indicates an increased rate of GSH usage and the depletion of tGSH pool as seen in the present results.

In conclusion, the concentration of GSH was generally decreased in KO Wfsl-deficient mice. A slight, but not statistically significant increase was seen in liver at young age. In HZ mice, statistically significant or minimal increases of tGSH were observed in the kidneys and pancreatic tissue at older age. The upregulation of GSH in the liver of 2-month-old KO mice is probably an attempt to control ER stress and depends on the expected expression of $W f s I$.

\section{Acknowledgements}

The authors wish to thank the undergraduate students in the Department of Biochemistry, University of Tartu, Ms. Mariin Rehe, Ms. Anu Viispert, Ms. Helen Raude, Ms. Alina Lebedeva and Mr. Oliver Arg for technical assistance. This research was supported by Institutional Research Funding (grant no. IUT20-42), The Center of Excellence for Genomics and Translational Medicine from the Estonian Ministry of Education and Science, and by the European Union through the European Regional Development Fund (project no. 2014-2020.4.01.15-0012). 


\section{References}

1. Strom TM, Hörtnagel K, Hofmann S, Gekeler F, Scharfe C, Rabl W, Gerbitz KD and Meitinger T: Diabetes insipidus, diabetes mellitus, optic atrophy and deafness (DIDMOAD) caused by mutations in a novel gene (wolframin) coding for a predicted transmembrane protein. Hum Mol Genet 7 : 2021-2028, 1998.

2. Inoue $\mathrm{H}$, Tanizawa $\mathrm{Y}$, Wasson $\mathrm{J}$, Behn $\mathrm{P}$, Kalidas $\mathrm{K}$, Bernal-Mizrachi E, Mueckler M, Marshall H, Donis-Keller H, Crock $\mathrm{P}$, et al: A gene encoding a transmembrane protein is mutated in patients with diabetes mellitus and optic atrophy (Wolfram syndrome). Nat Genet 20: 143-148, 1998.

3. Hofmann S, Philbrook C, Gerbitz KD and Bauer MF: Wolfram syndrome: Structural and functional analyses of mutant and wild-type wolframin, the WFS1 gene product. Hum Mol Genet 12: 2003-2012, 2003.

4. Takeda K, Inoue H, Tanizawa Y, Matsuzaki Y, Oba J, Watanabe Y, Shinoda K and Oka Y: WFS1 (Wolfram syndrome 1) gene product: Predominant subcellular localization to endoplasmic reticulum in cultured cells and neuronal expression in rat brain Hum Mol Genet 10: 477-484, 2001.

5. Fonseca SG, Ishigaki S, Oslowski CM, Lu S, Lipson KL, Ghosh R, Hayashi E, Ishihara H, Oka Y, Permutt MA and Urano F: Wolfram syndrome 1 gene negatively regulates ER stress signaling in rodent and human cells. J Clin Invest 120 744-755, 2010

6. Fonseca SG, Fukuma M, Lipson KL, Nguyen LX, Allen JR, Oka Y and Urano F: WFS1 is a novel component of the unfolded protein response and maintains homeostasis of the endoplasmic reticulum in pancreatic beta-Cells. J Biol Chem 280: 39609-39615, 2005

7. Urano F: Wolfram syndrome iPS cells: The first human cell model of endoplasmic reticulum disease. Diabetes 63: 844-846, 2014.

8. Walter P and Ron D: The unfolded protein response: From stress pathway to homeostatic regulation. Science 334: 1081-1086, 2011

9. Zeeshan H, Lee G, Kim HR and Chae HJ: Endoplasmic reticulum stress and associated ROS. Int J Mol Sci 17: 327, 2016.

10. Delaunay-Moisan A and Appenzeller-Herzog C: The antioxidant machinery of the endoplasmic reticulum: Protection and signaling. Free Radic Biol Med 83: 341-351, 2015.

11. Ellgaard L and Ruddock LW: The human protein disulphide isomerase family: Substrate interactions and functional properties. EMBO Rep 6: 28-32, 2005.

12. Cullinan SB, Zhang D, Hannink M, Arvisais E, Kaufman RJ and Diehl JA: Nrf2 is a direct PERK substrate and effector of PERK-dependent cell survival. Mol Cell Biol 23: 7198-7209, 2003.

13. Harding HP, Zhang Y, Zeng H, Novoa I, Lu PD, Calfon M, Sadri N, Yun C, Popko B, Paules R, et al: An integrated stress response regulates amino acid metabolism and resistance to oxidative stress. Mol Cell 11: 619-633, 2003.

14. Ballatori N, Krance SM, Notenboom S, Shi S, Tieu K and Hammond CL: Glutathione dysregulation and the etiology and progression of human diseases. Biol Chem 390: 191-214, 2009.

15. Luuk H, Plaas M, Raud S, Innos J, Sütt S, Lasner H, Abramov U, Kurrikoff K, Kõks S and Vasar E: Wfs1-deficient mice display impaired behavioural adaptation in stressful environment. Behav Brain Res 198: 334-45, 2009.

16. Tietze F: Enzymic method for quantitative determination of nanogram amounts of total and oxidized glutathione: Applications to mammalian blood and other tissues. Anal Biochem 27: 502-522, 1969.
17. Ursini F, Maiorino $M$ and Gregolin C: The selenoenzyme phospholipid hydroperoxide glutathione peroxidase. Biochim Biophys Acta 839: 62-70, 1985.

18. Carlberg I and Mannervik B: Glutathione reductase. Methods Enzymol 113: 484-90, 1985.

19. Geraghty P, Baumlin N, Salathe MA, Foronjy RF, D'Armiento JM and Armiento JM: Glutathione Peroxidase-1 suppresses the unfolded protein response upon cigarette smoke exposure. Mediators Inflamm 2016: 9461289, 2016.

20. Eletto D, Chevet E, Argon Y and Appenzeller-Herzog C: Redox controls UPR to control redox. J Cell Sci 127: 3649-3658, 2014.

21. Li S, Li X, Li YL, Shao CH, Bidasee KR and Rozanski GJ: Insulin regulation of glutathione and contractile phenotype in diabetic rat ventricular myocytes. Am J Physiol Hear Circ Physiol 292: H1619-H1629, 2007.

22. Tiedge M, Lortz S, Drinkgern J and Lenzen S: Relation between antioxidant enzyme gene expression and antioxidative defense status of insulin-producing cells. Diabetes 46: 1733-1742, 1997.

23. Tran PO, Parker SM, LeRoy E, Franklin CC, Kavanagh TJ, Zhang T, Zhou H, Vliet P, Oseid E, Harmon JS and Robertson RP: Adenoviral overexpression of the glutamylcysteine ligase catalytic subunit protects pancreatic islets against oxidative stress. J Biol Chem 279: 53988-53993, 2004.

24. Shang L, Hua H, Foo K, Martinez H, Watanabe K, Zimmer M, Kahler DJ, Freeby M, Chung W, LeDuc C, et al: $\beta$-Cell dysfunction due to increased ER stress in a stem cell model of wolfram syndrome. Diabetes 63: 923-933, 2014.

25. Tolman KG, Fonseca V, Dalpiaz A and Tan MH: Spectrum of liver disease in type 2 diabetes and management of patients with diabetes and liver disease. Diabetes Care 30: 734-743, 2007.

26. Furfaro AL, Nitti M, Marengo B, Domenicotti C, Cottalasso D, Marinari UM, Pronzato MA and Traverso N: Impaired synthesis contributes to diabetes-induced decrease in liver glutathione. Int J Mol Med 29: 899-905, 2012.

27. Ishihara $H$, Takeda $S$, Tamura $A$, Takahashi R, Yamaguchi $S$, Takei D, Yamada T, Inoue H, Soga H, Katagiri H, et al: Disruption of the WFS1 gene in mice causes progressive beta-cell loss and impaired stimulus-secretion coupling in insulin secretion. Hum Mol Genet 13: 1159-1170, 2004.

28. Noormets K, Kõks S, Muldmaa M, Mauring L, Vasar E and Tillmann V: Sex differences in the development of diabetes in mice with deleted wolframin (Wfs1) gene. Exp Clin Endocrinol Diabetes 119: 271-275, 2011.

29. Ibrahim HN and Hostetter TH: Diabetic nephropathy. J Am Soc Nephrol 8: 487-493, 1997.

30. Kashihara N, Haruna Y, Kondeti VK and Kanwar YS. Oxidative stress in diabetic nephropathy. Curr Med Chem 17: 4256-69, 2010.

31. Urata Y, Yamamoto H, Goto S, Tsushima H, Akazawa S, Yamashita S, Nagataki S and Kondo T: Long exposure to high glucose concentration impairs the responsive expression of gamma-glutamylcysteine synthetase by interleukin-1beta and tumor necrosis factor-alpha in mouse endothelial cells. J Biol Chem 271: 15146-15152, 1996.

32. Catherwood MA, Powell LA, Anderson P, McMaster D, Sharpe PC and Trimble ER: Glucose-induced oxidative stress in mesangial cells. Kidney Int 61: 599-608, 2002.

33. Szymonik-Lesiuk S, Czechowska G, Stryjecka-Zimmer M, Słomka M, Maldro A, Celiński K and Wielosz M: Catalase, superoxide dismutase, and glutathione peroxidase activities in various rat tissues after carbon tetrachloride intoxication. J Hepatobiliary Pancreat Surg 10: 309-315, 2003

34. Jurczuk M, Moniuszko-Jakoniuk J and Brzóska MM: Involvement of some low-molecular thiols in the peroxidative mechanisms of lead and ethanol action on rat liver and kidney. Toxicology 219: 11-21, 2006. 